Musées, Patrimoine et Culture scientifiques et techniques

179 | 2018

septembre-octobre 2018

\title{
Une exposition drôlement juste aux musées \\ Gadagne
}

Claire Déglise, Jennifer Aucagne et Xavier de la Selle

\section{OpenEdition \\ Journals}

Édition électronique

URL : http://journals.openedition.org/ocim/2802

DOI : $10.4000 /$ ocim.2802

ISSN : 2108-646X

Éditeur

OCIM

Édition imprimée

Date de publication : 1 septembre 2018

Pagination : $42-49$

ISSN : 0994-1908

Référence électronique

Claire Déglise, Jennifer Aucagne et Xavier de la Selle, « Une exposition drôlement juste aux musées Gadagne ", La Lettre de I'OCIM [En ligne], 179 | 2018, mis en ligne le 01 septembre 2019, consulté le 07 septembre 2019. URL : http://journals.openedition.org/ocim/2802 ; DOI : 10.4000/ocim.2802

Ce document a été généré automatiquement le 7 septembre 2019

Tous droits réservés 


\title{
Une exposition drôlement juste aux musées Gadagne
}

\author{
Claire Déglise, Jennifer Aucagne et Xavier de la Selle
}

Statuette de grand prêtre d'un culte de la nature, portant le couvre-chef et la barbe, apanages des classes dominantes, et tenant un gobelet à libations au fond percé : une partie du breuvage s'écoulait ainsi en offrande à la terre nourricière. Terre cuite peinte. Exposition Futur antérieur. Trésors archéologiques

du 21 e siècle après J.-C., 2002

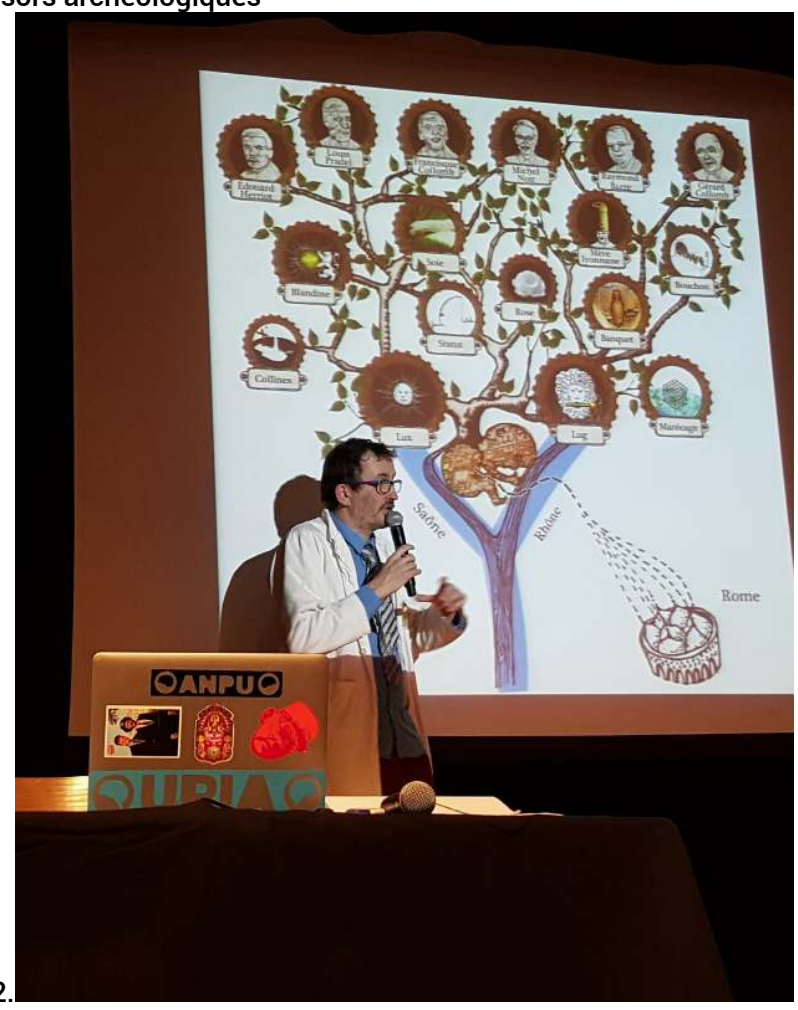

(c) Fibbi-Aeppli/Musée romain de Lausanne-Vidy 


\section{Mettre la ville sur le divan}

1 Au musée d'histoire de Lyon, une démarche originale a été adoptée pour proposer aux visiteurs une exposition consacrée à l'urbanisme la ville. Afin de donner à comprendre la ville contemporaine et les mutations qui l'ont façonnée, nous avons choisi de nous tourner vers le collectif artistique de l'Agence nationale de psychanalyse urbaine (ANPU), pour nous aider à écrire le concept et le scénario de l'exposition... puis de fil en aiguille pour travailler ensemble sur toute la chaîne de l'exposition, les contenus et la programmation associée.

2 Après plusieurs mois de travail collectif, de frictions et d'osmose, est née l'exposition Lyon sur le divan. Les métamorphoses d'une ville. À l'image de la double entrée du titre, le visiteur est entraîné dans une relecture teintée d'humour et de sérieux des grandes et petites métamorphoses de la ville, et est invité à se sentir plus concerné par sa fabrique.

3 À l'heure où nous écrivons cet article, l'exposition n'a pas encore fermé ses portes. Nous en profitons pour faire un premier partage d'expériences en interrogeant notre pratique par le prisme de l'humour dans l'exposition.

4 Pourquoi ce choix dans ce musée et avec ces as « de l'humour absurde » et du « farfelu poétique »? Comment la rencontre a-t-elle pu se faire, puis se matérialiser dans l'exposition et dans la programmation associée ? Au final, quel savoureux mélange d'humour et de sérieux se crée?

\section{De la licence poétique}

5 À bien des égards, Lyon sur le divan est une exposition-manifeste. Pour l'équipe du musée, elle témoigne de la volonté d'être davantage un musée de ville qu'un musée d'histoire. Par sa dimension expérimentale, elle veut explorer la possibilité pour le musée de retrouver sa vocation originelle, celle de donner les clés de lecture du territoire et permettre aux habitants de comprendre leur ville, en se situant dans l'espace et le temps. Il est apparu assez rapidement que la simple monstration de données historiques, de savoirs techniques et d'objets illustratifs ne suffirait pas, non seulement à séduire les visiteurs, mais surtout à permettre une appropriation active du sujet urbain. Plutôt que présenter frontalement des contenus ordonnés selon un plan scientifique, l'idée était donc d'abord de construire la scénarisation du propos. Mettre en récit la question potentiellement aride de l'urbanisme ne constitue pas simplement un habillage formel pour rendre les choses plus distrayantes, mais représente une véritable démarche de médiation. Cette démarche introduit une dimension fictionnelle pour ouvrir l'imaginaire des visiteurshabitants, leur permettre de se projeter eux-mêmes dans l'exposition et de s'y sentir à l'aise au point d'y apporter leur contribution et leur expérience urbaine. 
Laurent Petit, responsable de l'ANPU, lors de sa conférence introductive à la psychanalyse urbaine, le 18 novembre 2017

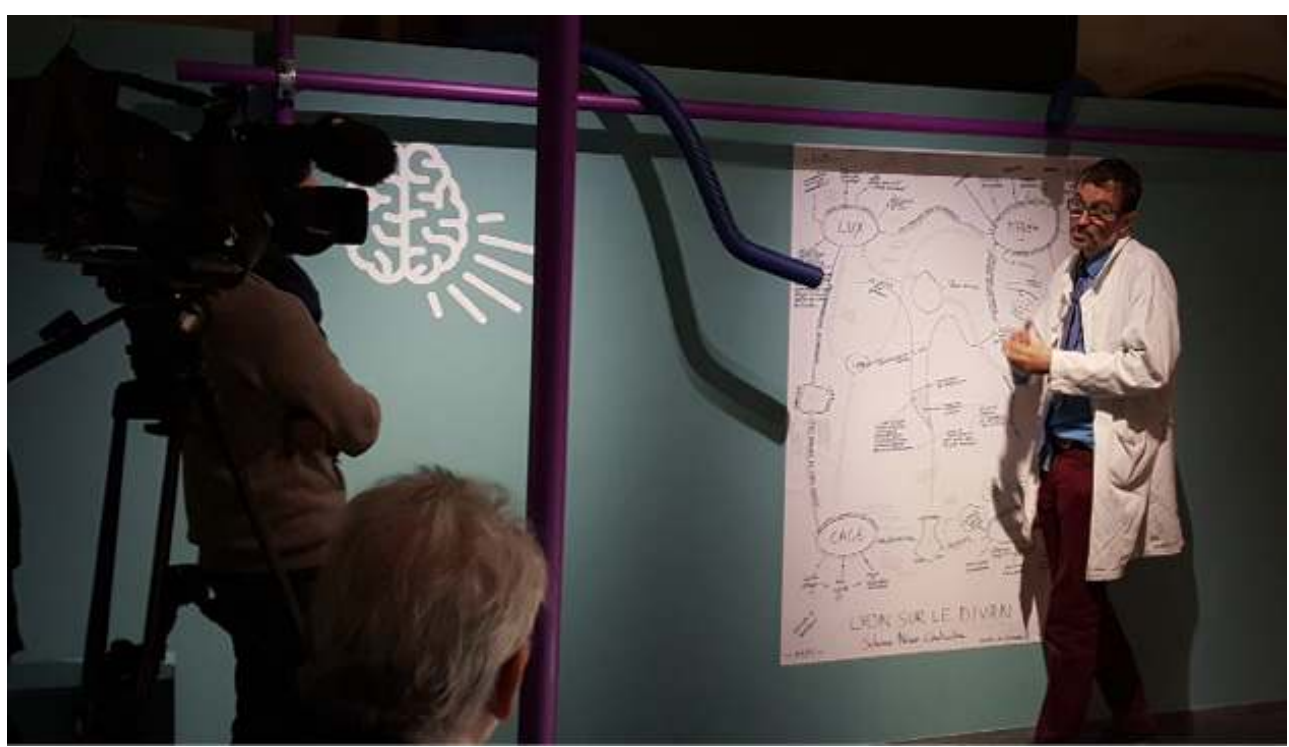

(C) Musées Gadagne

6 L'autre fondement de cette approche repose sur l'intégration d'un partenaire artistique, au risque du dérapage incontrôlé, de l'attitude indisciplinée et du regard critique sur les politiques urbaines. C'est ici qu'intervient l'enjeu du positionnement du musée de ville, dans le rôle d'espace de débat et d'expressions plurielles. Dans cette entreprise délicate, où l'exposition met en scène aussi bien l'évocation historique que l'actualité des projets urbains, le recours à la poésie et à l'humour peut donner au musée une forme de licence poétique.

La partie de l'exposition consacrée au corps urbain et au cerveau.

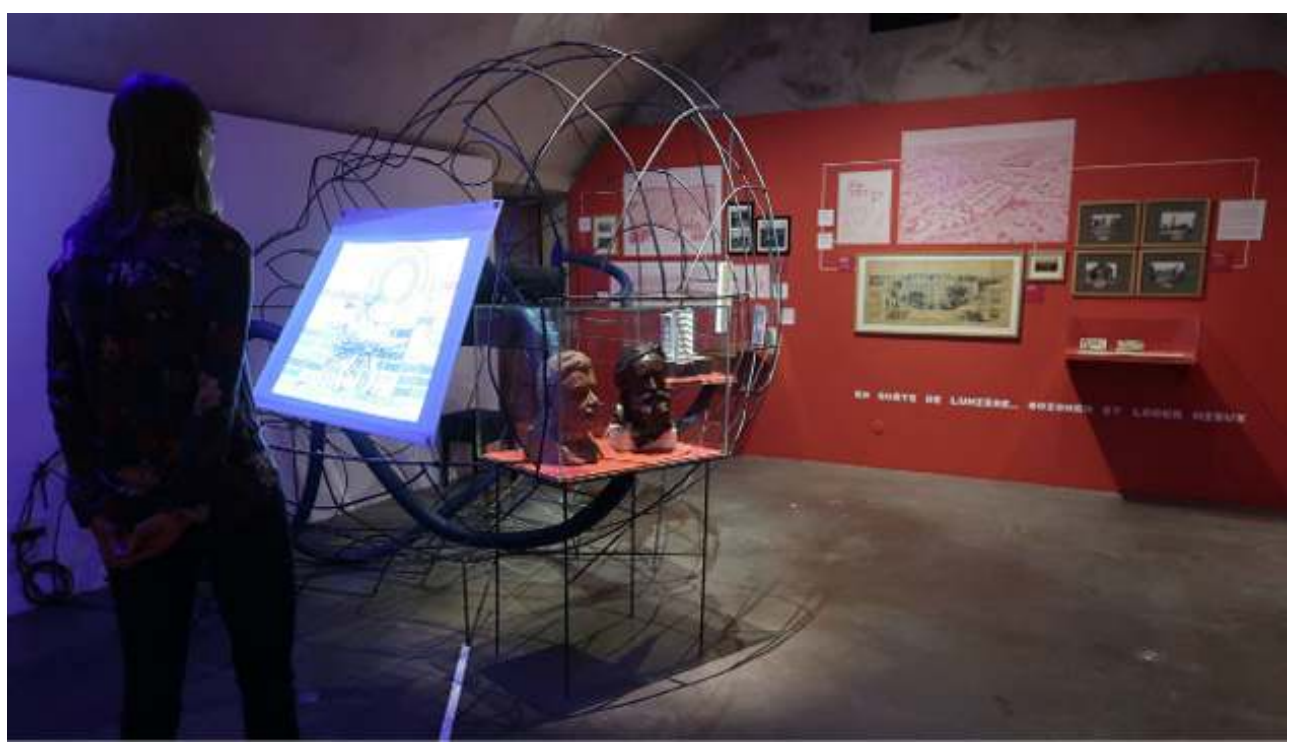

(c) Musées Gadagne 


\section{Quel humour ? Pourquoi l'ANPU ?}

7 À l'issue de débats au sein de l'équipe projet, nous avons décidé de faire appel à l'ANPU. Ce collectif d'artistes, né en 2007, est l'inventeur d'une nouvelle science déstabilisante poétique et farfelue : la " psychanalyse urbaine».

8 Trois raisons ont motivé ce choix :

9 - leur force d'artistes polymorphes, à la fois rompus aux codes et langages de l'urbanisme (plusieurs d'entre eux sont architectes de formation), mais aussi agiles sur scène et dans la rue pour développer leur propre poétique de la ville, bref des " geo-artistes ", grâce à qui, selon Luc Gwiazdzinski et Lisa Pignot, "s'inventent in situ d'autres imaginaires, représentations et procédures qui participent à la fabrique de la ville et de l'urbanité $»^{1}$;

10 - leur capacité à nous aider à prendre de la distance avec notre sujet : en faisant parler notre territoire grâce à leur protocole bien rodé, et en révélant son diagnostic sur un mode débonnaire, nous avons cherché à libérer les imaginaires, déplacer notre regard et celui du visiteur, faire rire pour ensuite faire réfléchir ;

11 - notre volonté de rester en cohérence avec une de nos réflexions de départ concernant un nouveau public cible que nous souhaitions toucher avec cette exposition : l'actif urbain néophyte en urbanisme, qui pratique donc la ville au quotidien. Nous avons misé sur son envie de mieux connaître cette ville, d'en parler, voire de se sentir concerné par ses transformations. Cela ne nous a pas empêché en parallèle de continuer à traiter le sujet en profondeur avec un comité scientifique, un catalogue, et de chercher à maintenir le lien avec nos publics habituels.

12 En faisant appel à l'ANPU, c'est donc tout un monde que nous avons fait entrer au musée. Ce monde se caractérise par :

13 - son vocabulaire inspiré de la "psychanalyse de comptoir », où le ça, le moi et le surmoi se transforment en « ça», « toit » et « surtoit»;

14 - ses innombrables acronymes parodiant la tendance lourde des urbanistes à utiliser des sigles abscons. Régulièrement l'équipe détecte sur les « territoires patients " un PNSU, Point Névro Stratégique Urbain, « un lieu symbolique où viennent se concentrer toutes les névroses urbaines » sur lequel elle prescrit un TRU (Traitement Radical Urbain), ou un TRA (Traitement Radical Architectural) voire un TRC (Traitement Radical Catharsistique) « afin que le territoire patient parvienne à son plein épanouissement d' ici 30 ans si tout se passe bien ou d' ici 40 ans si le territoire patient est particulièrement atteint »;

15 - son site Internet (www.anpu.fr) qui décrit à grand renfort de citations mi-sérieuses miloufoques sa méthodologie avec des «step " à franchir pour arriver au nirvana urbain. Une méthodologie qui s'appuie sur les fameux « 8 commandements » de la psychanalyse urbaine : "élaborés par James Lawson lors d'un pèlerinage à Vienne au numéro 9 de la Berggasse, le lieu où résida Sigmund Freud jusqu'à son exil forcé à Londres en 1938, ces huit commandements constituent incontestablement la base théorique et philosophique de la psychanalyse urbaine... L'analyse urbaine se fait toujours par tâtonnements progressifs, il faut que la ville accepte de se faire tâter un peu partout, même à des endroits qui peuvent paraître a priori gênants voire indélicats... »; 
Opération divan à la Part-Dieu en décembre 2016

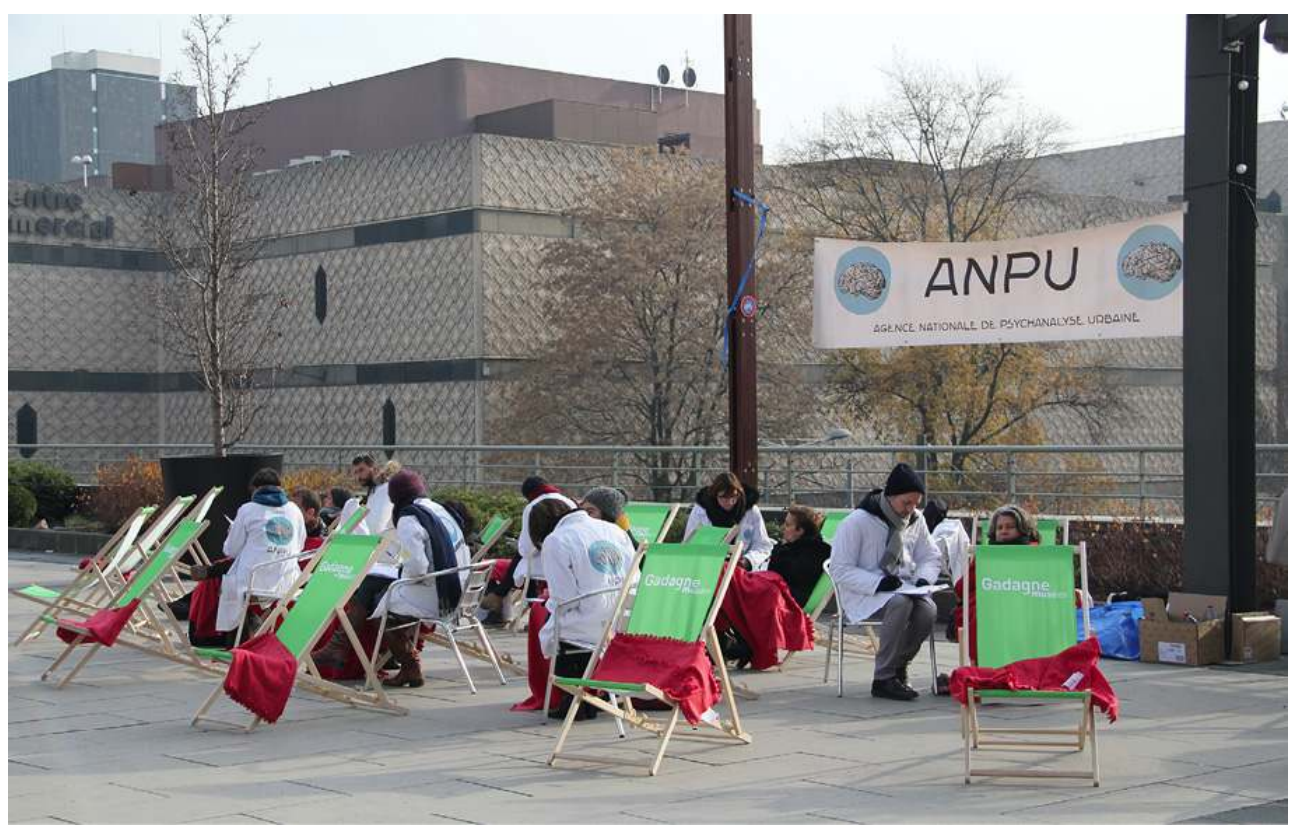

(c) Musées Gadagne

- et bien sûr son ambassadeur Laurent Petit, grand bonhomme à la blouse blanche, " champion du monde de la manipulation des mots ", auteur de leur «Bible " : L'Introduction à la psychanalyse du monde entier ${ }^{2}$, sorte de synthèse brinquebalante entre Don Quichotte, Monsieur Hulot, Sigmund Freud, Emil Cioran, Jacques Lacan, le professeur Burp de Gotlib et Maître Capello : « après une brève carrière d'ingénieur, Laurent Petit se lance dans le monde merveilleux du spectacle en étant tout d'abord jongleur, puis clown de supermarché. La rencontre avec Éric Heilmann et ses travaux sur les liens entre Mickey la souris et Michel-Ange va lui permettre de jeter les bases d'un genre nouveau, le spectacle para-scientifique, genre où le vrai et le faux se mélangent tellement bien que le public finit par en perdre son latin ».

Mélange de farfelu et sérieux, ces conférences jouent avec l'ambiguïté avoue Laurent Petit : « on n'est ni dans un discours politique, ni moralisateur. C'est au spectateur de décider s'il assiste à un spectacle ou à une conférence». Il souligne par ailleurs « mais souvent par l'humour on fait passer plus de messages qu'on ne le pense ».

18 Entre avril et septembre 2017, le temps de la gestation du projet, nous avons d'ailleurs commencé à plonger nos visiteurs dans cet univers avec une installation teaser au sein des futurs espaces d'exposition. Autour du « bureau du directeur-psychanalyste » et de quelques écrans présentant la future exposition et les étapes déjà réalisées, les visiteurs ont été invités à fouler l'immense bâche reprenant les " 8 commandements » de la psychanalyse urbaine pour finir par s'allonger dans des divans-transat afin de remplir des " questionnaires chinois » et dessiner un portrait de leur ville. «Pour ne pas tomber dans les histoires de crottes de chien qui ne nous intéressent pas, on doit déclencher de la poétique ", préconise l'ANPU. Alors, si Lyon était un fruit? Ou encore un animal ? Ou une chanson? Les réponses sont parfois surprenantes et passionnantes. En voici quelques extraits : si Lyon était un légume ou un fruit? « une citrouille! Grosse, lourde et pesante mais... pouvant se transformer en carrosse ", " une petite pomme, jaunâtre à cause des gaz de l'autoroute A7. Elle voudrait être Big Apple mais n'est encore qu'une pomme d'amour ». 


\section{Comment la rencontre entre humour et sérieux s'est- elle matérialisée dans l'exposition?}

19 Une fois passé l'enthousiasme des débuts, les tâtonnements furent nombreux, tant pour l'ANPU qui collaborait pour la première fois avec un musée à l'écriture d'une exposition que pour nous. Rompus au travail avec des comités scientifiques, nous n'avions jamais fait appel à un commissariat artiste encore moins humoristique pour décaler nos habitudes d'écriture d'exposition.

20 Afin d'écrire le scénario de l'exposition, outre le travail de documentation et les réunions avec le comité scientifique, nous avons décidé de nous appuyer sur le protocole bien rodé et fécond de l'ANPU pour arriver au diagnostic du territoire. Une série d'opérations divan lancées dans l'espace public à la rencontre des habitants et de leurs ressentis de la ville, accompagné d'une trentaine d'entretiens avec des experts de toutes natures (du géographe au patron de café, du sociologue à l'ancien élu). Après avoir livré un premier diagnostic relativement sage et historique, Laurent Petit réussit ensuite à appliquer quelques-uns de ses outils habituels sur la métropole bimillénaire :

21 - l'arbre mytho-généalogique de la ville-patiente qui se base sur son vécu social, ses légendes urbaines et ses traumatismes historiques. Voici quelques exemples d'« ancêtres ancestraux » détectés sur Lyon : son père fondateur, l'Empire romain qui « en imposant à Lyon de devenir dès sa naissance urbaine la capitale des Gaules... a confié à la ville naissante des responsabilités sans doute écrasantes... il est à se demander... si un schéma parental aussi lourd à porter n'a pas initié le rapport complexe qu'entretient la ville avec la notion de capitale et si Lyon ne souffre pas aujourd'hui d'être une capitale contrariée "; ses parents géologiques, le Rhône et la Saône " aux tempéraments diamétralement opposés et sans doute à l'origine du côté insaisissable d'une ville qui est à la fois... une ville de l'ombre, une ville de lumière, une ville molle et une ville dynamique, une ville rebelle et une ville conformiste... ».

Une fresque collaborative est proposée en fin d'exposition.

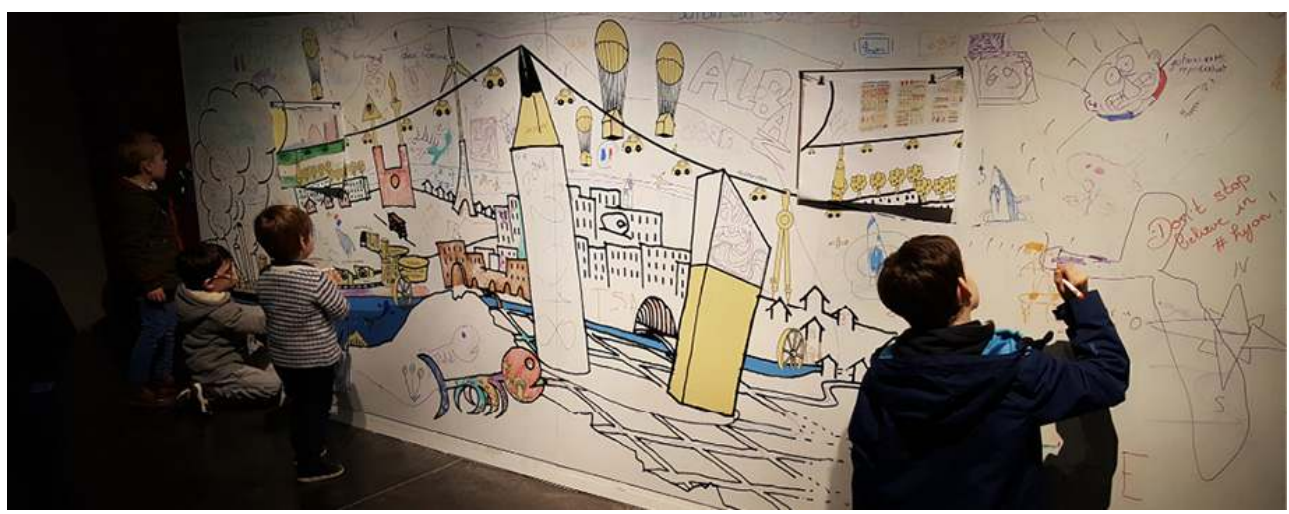

(c) Musées Gadagne

22 - le SNC (schéma névro-constructeur), qui selon ses concepteurs est LA « technique d'analyse permettant de décrypter les névroses du territoire patient via toutes sortes de recoupements sémantiques inspirés par le grand Jacques, alias Jacques Lacan ». Sur Lyon, le schéma se révèle comme sur les autres villes, génialement labyrinthique et incompréhensible au premier regard. Pourtant selon son auteur cela résume des 
évidences et des tensions psychiques qui vont interférer durant toute l'histoire de la ville Anpusienne ? En concevant une exposition résolument Anpusienne avec des petites capsules scientifiques, ou bien en imaginant une exposition scientifique agrémentée de commentaires artistiques? Nous avons opté finalement pour une ligne médiane : faire coexister les deux discours, pour les faire résonner dans l'esprit des visiteurs afin qu'ils puissent en retour s'exprimer. Selon la typologie définie par Serge Chaumier, il s'agit d'une « muséographie dissociée »".

Installation collaborative à destination des familles sur la ville végétale, Week-end " ma ville rêvée " 17 mars 2018

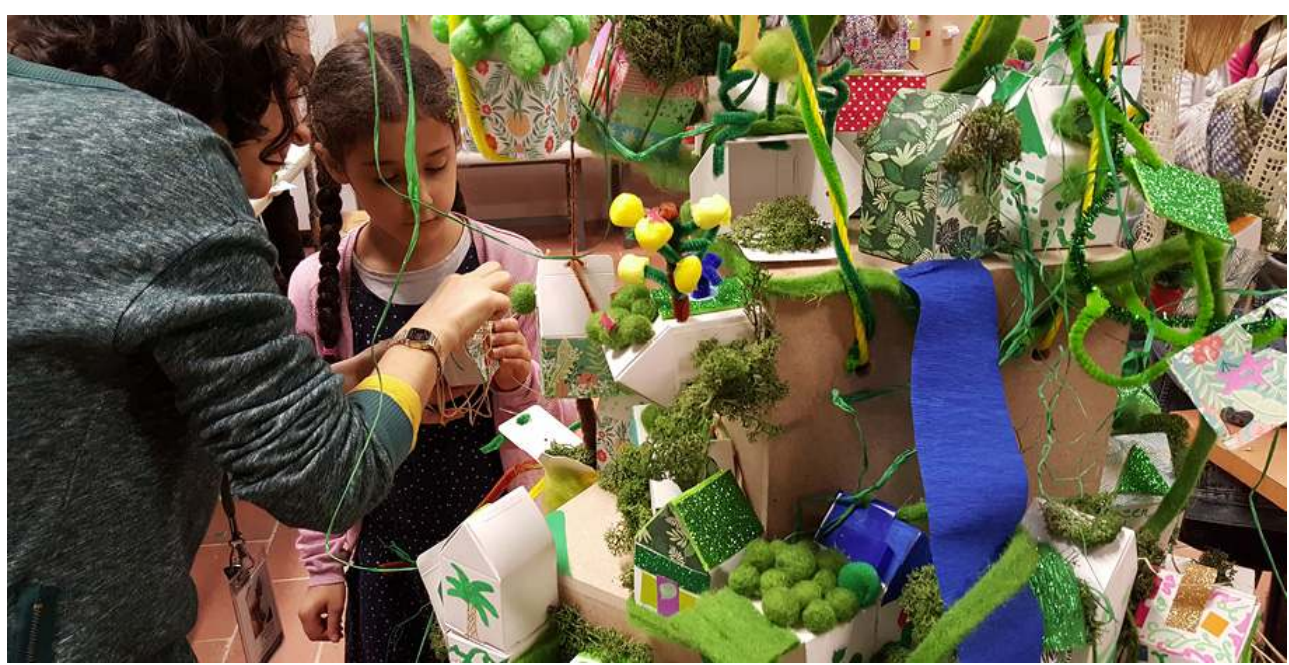

(c) Musées Gadagne

Autour d'une introduction et d'une conclusion à tonalité ANPUsiennes, l'une s'appuyant sur l'arbre mytho-généalogique, l'autre sur le SNC et la projection de Lyon " guérie » en 2118, le cœur du discours s'est ensuite déployé autour de 4 thèmes, telles 4 séances de psychanalyse, faisant alterner le récit allégorique et humoristique du psychanalyste avec une analyse scientifique venant confirmer ou nuancer ce thème : l'extension urbaine, en lien avec l'image d'une marée urbaine et de poussées de croissances tous azimuts ; la

La Lettre de l'OCIM, 179 | 2018 
couture urbaine, en lien avec les nombreux raccordements opérés sur le tissu urbain ; l'hygiénisme, en lien avec l'image d'une ville de marécages et en quête de lumière(s) ; le marketing urbain et la modernisation de la ville, en lien avec l'image de la surnarcissisation d'une grande métropole.

En réponse à ce scénario à la fois fort et structuré, l'équipe scénographique pilotée par Le Muséophone et Inclusit Design, a proposé une scénographie résolument intrigante. Lyon, personnifiée, se raconte sur le divan : face au visiteur, un grand corps urbain fait de tiges métalliques et de tubes se déploie à travers les espaces.

Sur cette ville, au début de chaque partie un cerveau loufoque incarne le psychanalyste urbain et projette ses diagnostics allégoriques et sonores à travers des textes lus de Laurent Petit et des dessins de Charles Altorffer. En écho, un mur d'analyses scientifiques répond aux interrogations du cerveau en s'appuyant sur une présentation comparative de différentes œuvres et documents. Enfin dernier temps, des tribunes «Et pour vous?» permettent aux habitants-visiteurs de s'exprimer sur le sujet. À l'appui de ce choix muséographique, les écritures se sont organisées. Dans un souci de relier le tout, les titres des parties sont courts afin de résonner tant du côté humoristique que du côté urbanistique : rajeunissement, croissance, coutures, santé.

\section{Créer les conditions optimales pour découvrir l'exposition}

29 En médiation, l'humour est un levier qui permet de dédramatiser une situation, un sujet, un contenu et de faire tomber les défenses. Aussi tout naturellement, intégrer des rendezvous décalés et humoristiques dans la programmation de l'exposition, s'est imposé à l'équipe du service des publics pour prolonger le propos muséographique.

30 L'équipe en concertation avec les services exposition et communication a imaginé deux temps forts qui ont rythmé la durée de l'exposition. Deux week-ends festifs en début d'exposition et à mi-parcours proposaient des activités pour permettre au visiteur de rentrer dans l'univers de l'ANPU, avec des interventions menées par les artistes :

31 - des conférences-spectacles : sur le premier temps festif, en début d'exposition, une démonstration loufoque présentait la méthodologie appliquée à cette science particulière inventée par l'ANPU inspiré du traditionnel format des conférences Powerpoint. Dans le second temps proposé, une forme théâtrale créée à partir des contributions des visiteurs issues des différents espaces participatifs présents dans l'exposition faisait apparaitre une cartomancienne lisant l'avenir de la ville ;

32 - une balade urbaine improvisée d'un lieu clé de la ville, le centre d'échanges Perrache, (PNSU) point névralgique de tensions urbaines s'inventait sous la forme d'une promenade poétique pilotée par un artiste marcheur, se définissant comme un " urbaniste grands pieds, urbaniste grand prix d'urbanisme de l'académie d'architecture, urbaniste gonzo, street jockey provençopolitain, éminence grise de l'agence nationale de psychanalyse urbaine marseillologue buccorhodanéologiste, guide tous terrains spécialisé dans l'attention pour les situations construites, cartographe et montreur d'ours en béton ".

Cette offre artistique était complétée par des temps de médiation présentielle en salles par l'équipe des musées Gadagne afin d'aider les visiteurs à appréhender le parcours de visite et à se laisser porter par la démarche des psychanalystes urbains après avoir 
succombé à la dégustation d'un bonbon « cerveau ». Décryptage des allégories imaginées par l'artiste Charles Altorffer présentes dans les collections, présentation des dispositifs « cerveau » ou orientation vers les tribunes.

Slogans inventés par les visiteurs dans la dernière partie consacrée au marketing urbain et à la surnarcissisation de la ville et postés sur Instagram.
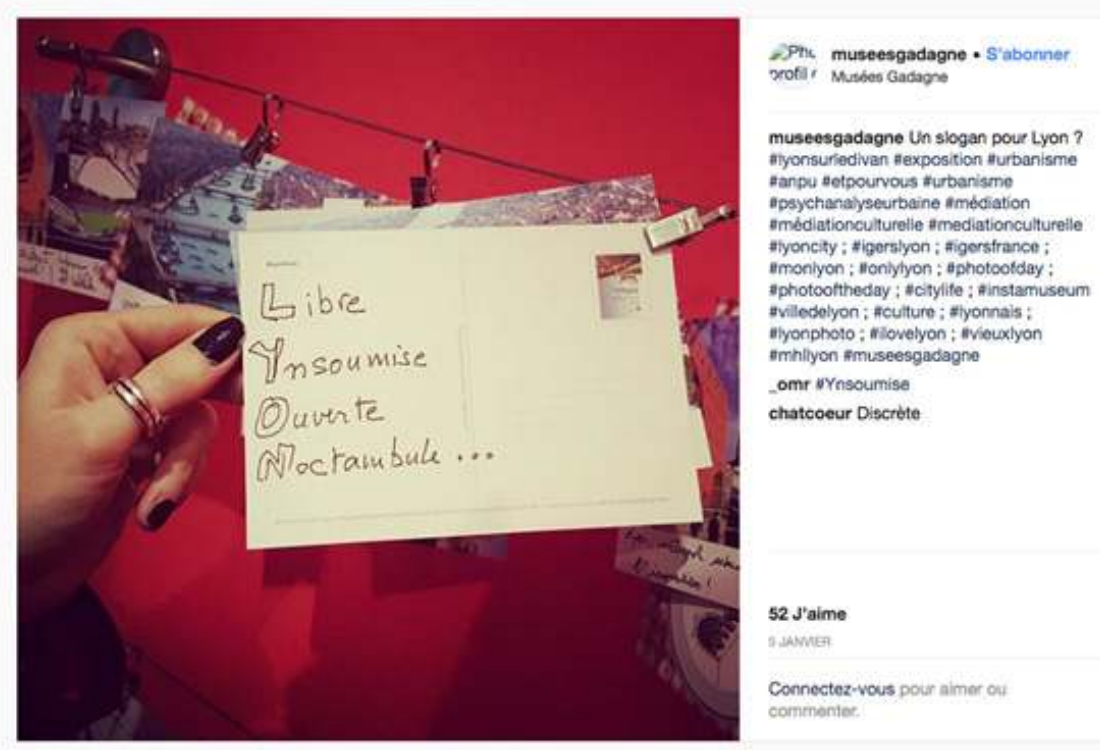

A PROPOS DENOUS SUPPORT BLOC PRESSE API EMPLOIS CONFDENTLUTtE CONDINONS AEPERTOIRE PROFLS HASHTAOS Lavaue

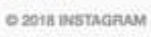

(c) Musées Gadagne

été construites et animées par les acteurs et experts qui nous ont accompagnés tout au long de la préparation de l'exposition : Agence d'urbanisme, Conseil d'architecture, d'urbanisme et de l'environnement, librairie Archipel. Elles ont permis de toucher un nouveau public, à la fois curieux et concerné par ces questions, assidu à chacune de ces rencontres;

37 - des activités plus participatives ou destinées aux familles, des partenariats avec d'autres acteurs de la ville, comme des ateliers pour créer et imaginer sa ville rêvée, la ville de demain ou encore un concours Instagram sur « votre métamorphose de Lyon préférée ». 
tte oscillation entre humour et sérieux a permis à chaque visiteur de pouvoir choisir dans la programmation l'activité qui lui convenait le mieux. Avec la carte de l'humour, le public a pu s'exprimer plus facilement sur sa ville dans les espaces dédiés, chasser ses a priori et ainsi à ce s'approprier davantage sa ville et le musée. Si bien sûr, des visiteurs ont été surpris voire choqués, l'engouement global et la réceptivité à la démarche " ANPUsienne " ont pu être observé à plusieurs reprises, notamment dans la tribune " cartes postales » consacrée à l'écriture de slogans sur la ville, et dans l'espace conclusion où se mêlaient créativité et amusement sur la fresque collaborative proposée par l'ANPU «Lyon en 2118 ».

Enfin, ce que nous avons pu apprendre ou développer avec cette exposition et cette collaboration avec l'ANPU (opérations divan, tribunes et interpellations des habitants) va être réutilisé dans le futur réaménagement du parcours permanent du musée d'histoire.

\section{Quel est le type d'humour créé ?}

Si l'on peut parler d'humour dans cette exposition, tant dans sa présentation que dans sa programmation, il n'est en fait jamais frontal.

En premier lieu, il s'appuie plutôt sur un jeu poétique de la personnification de la ville décliné dans les allégories dessinées de Charles Altorffer et dans la structure scénographique. Ce jeu et cette structure viennent en écho au jeu littéraire du portrait chinois de la ville proposé aux habitants dans les Opérations divan et l'installation teaser (plus de 1000 questionnaires remplis). Ce jeu repose lui-même sur le vaste dispositif, proche du canular 5, patiemment élaboré par l'ANPU depuis 2007, autour de l'invention de la psychanalyse urbaine, cette nouvelle science fictive, qui sème le trouble chez le visiteur et encore plus chez le spectateur lorsqu'il voit Laurent Petit entrer en scène. Elle joue sur l'ultra sérieux du scientifique psychanalyste en blouse blanche qui manie concepts à tour de bras (le sur-moi, le ça mais aussi syndromes et autres névroses), et parodie « la tendance lourde des urbanistes à utiliser des sigles abscons». Cela prend tout son sens lorsque Laurent Petit nous donne sa définition du bouffon :

"Le rôle du bouffon n'est pas seulement de se moquer du roi (et avec lui de la ville, la mairie, le théâtre, le pouvoir), des personnes ou de l'institution, c'est de semer la confusion dans la tête des gens qui viennent l'écouter pour démonter leurs certitudes, leurs repères et les vérités auxquelles ils croient. L'idée est de déstabiliser l'assistance, non pas en remplaçant une vérité par une autre, mais plutôt en lui suggérant qu'il n'y a pas de vérité et qu'il n'y en aura sans doute jamais. La posture du bouffon n'est donc surtout pas celle du militant, il n'est pas là pour faire la révolution; il est là pour semer la confusion dans la tête des gens avec le secret espoir de leur ouvrir des portes qui donnent sur l'infini ».

Subtilement ensuite c'est dans les rapports d'analyses déclamés dans l'exposition que peuvent se déployer la manipulation des mots et le batifolage dans les champs lexicaux de la psychanalyse : double-sens, second degré, mots valises, lapsus et calembours se glissent au détour des phrases dites avec sérieux et aplomb: 


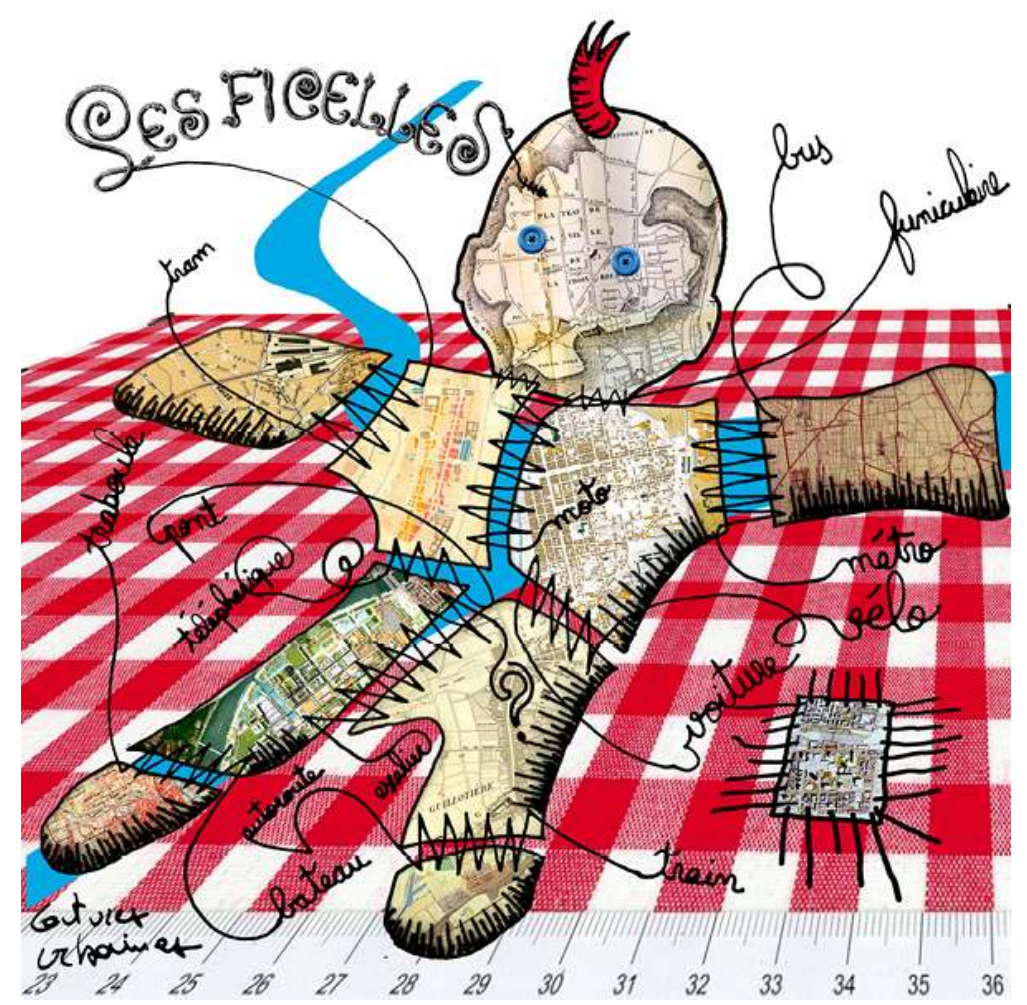

(c) ANPU/Charles Altorffer

- Allégorie $n^{\circ} 4$ : Une seconde jeunesse : «cette tendance narcissique avérée, déjà présente dans l'étrange habitude qu'a prise la ville de tapisser ses murs avec des grandes fresques à son effigie, n'est-elle pas en train d'aller trop loin ? Quand on se penche par exemple sur le projet d'agrandissement du quartier de la Part-Dieu, n'y a-t-il pas danger de voir apparaitre deux PartDieu (Depardieu) ?»;

- Allégorie $n^{\circ} 2$ : une ville (re)cousue de fils blancs : « Lyon s'affirme comme une grande spécialiste de la haute couture urbaine, une tradition qui remonte sans doute à l'histoire des soyeux et cette capacité à filer doux, à être à la fois inventif et coopératif face aux défis que suppose le développement d'une ville";

46 - Schéma nevro-constructeur et conclusion : « le risque de voir une Marée Urbaine se déployer à l'infini planera encore longtemps, mais il y a fort à parier que Lyon saura gérer ces différentes pulsions, en s'appuyant sur une longue pratique du con-sensuel; ce que ne fait, inconsciemment bien sûr, que confirmer la libido exacerbée d'une ville fort capable d'être toujours plus créative et encore plus illuminée dans les décennies voir les siècles à venir ».

Et c'est ainsi que l'ANPU met à jour des réalités tangibles qu'elles soient aberrations architecturales ou inhibitions larvées. Tout ce " mic-mac drolatique » fait surgir des vérités de l'inconscient collectif de cette cité bien campée entre crises de capitale contrariée et tendances narcissiques avérées. 


\section{Conclusion}

L'évaluation du projet sera mise à profit par le musée d'histoire de Lyon dans le cadre du renouvellement global de son parcours permanent. Mais les premières analyses et les réactions des publics nous encouragent à continuer à travailler dans ce sens. Tenter d'associer données factuelles et productions symboliques, faits historiques et regards poétiques n'est pas sans risques : le musée peut brouiller les messages et remettre en cause le «statut d'autorité » qu'on attend de lui. Oser introduire l'humour dans son langage devient alors un parti-pris : celui d'abandonner une pseudo-neutralité, d'assumer un discours, de le proposer à ses visiteurs sans se prendre trop au sérieux, et finalement d'essayer d'être « drôlement juste ».

\section{NOTES}

1. Gwiazdzinski, L. et Pignot, L. Les Géo-artistes : nouvelles dynamiques pour la fabrique métropolitaine, L'Observatoire. Revue des politiques culturelles, ${ }^{\circ} 48,2016$, pp. 19-31.

2. Petit, L. La Ville sur le divan, introduction à la psychanalyse urbaine du monde entier. Éditions La Contre Allée, 2013.

3. "Psychanalyser une ville comme Lyon n'a pas été de tout repos. Déjà techniquement parlant, pour une ville de cette taille sur un divan assez large et assez long pour qu'elle s'y installe, ne serait-ce qu'un court instant pour nous raconter sa vie était loin d'être évident... ». Extrait du rapport d'analyse issu du catalogue d'exposition : Lyon sur le divan. Les métamorphoses d'une ville. Éditions Libel, 2017.

4. Chaumier, S. Traité d'expologie. Les écritures de l'exposition. La Documentation française, 2012 p. 90. « En étant spatialement mis à distance, les objets de croyances, symboliques et les faits de science sont en possible dialogue, mais sans se mélanger. Un fil conducteur est proposé, et deux parcours peuvent même se décliner à partir de là».

\section{RÉSUMÉS}

Réalisée par les musées Gadagne, l'exposition Lyon sur le divan propose un regard décalé sur l'architecture et les métamorphoses de la ville grâce à un dispositif scénographique mêlant références sérieuses, parodie et humour. Les responsables du projet montrent comment ce choix audacieux peut décontenancer le visiteur mais aussi l'aider à s'approprier des contenus pour finalement mieux appréhender la ville. 
INDEX

Mots-clés : claire.deglise@mairie-lyon.fr

\section{AUTEURS}

CLAIRE DÉGLISE

Chargée des expositions aux musées Gadagne

JENNIFER AUCAGNE

Chargée de publics aux musées Gadagne

\section{XAVIER DE LA SELLE}

Directeur des musées Gadagne 